\title{
Supplement
}

\section{A multi-scale comparison of modeled and observed seasonal methane cycles in northern wetlands}

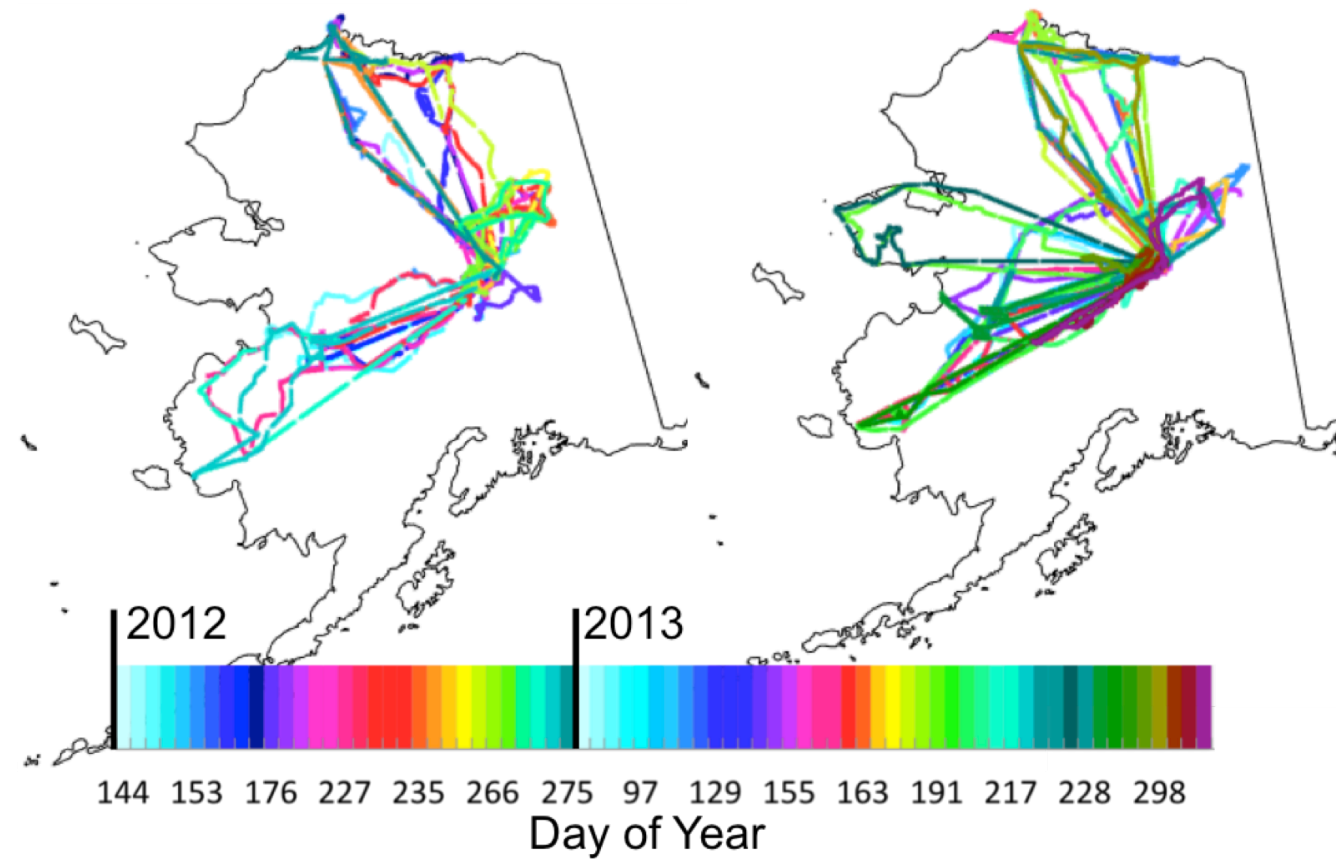

Fig. S1. Flight tracks with valid $\mathrm{CH}_{4}$ mole fractions during the CARVE 2012 and 2013 campaigns. There were 31 flight days in 2012 and 43 flight days in 2013 (Supplement Table S2). 


\begin{tabular}{|c|c|c|c|c|}
\hline Site Name & $\begin{array}{l}\text { Site Location } \\
\text { (Lat, Lon) }\end{array}$ & Citation & $\begin{array}{l}\text { Measurement } \\
\text { Method }\end{array}$ & Data years \\
\hline Ruoergai, China & $32^{\circ} 47^{\prime} \mathrm{N}, 102^{\circ} 32^{\prime} \mathrm{E}$ & Ding et al. (2004) & $\mathrm{SC}$ & 2001,2002 \\
\hline Mississippi, USA & $34^{\circ} 24^{\prime} \mathrm{N}, 89^{\circ} 50^{\prime} \mathrm{W}$ & Koh et al. (2009) & $\mathrm{SC}$ & 2005,2006 \\
\hline Michigan, USA & $42^{\circ} 27^{\prime} \mathrm{N}, 84^{\circ} 01^{\prime} \mathrm{W}$ & $\begin{array}{l}\text { Shannon and White } \\
\text { (1994) }\end{array}$ & $\mathrm{SC}$ & $\begin{array}{l}1991,1992, \\
1993\end{array}$ \\
\hline Minnesota, USA & $47^{\circ} 32^{\prime} \mathrm{N}, 93^{\circ} 28^{\prime} \mathrm{W}$ & Clement et al. (1995) & $\mathrm{SC}$ & 1991,1992 \\
\hline Minnesota, USA & $47^{\circ} 32^{\prime} \mathrm{N}, 93^{\circ} 28^{\prime} \mathrm{W}$ & Dise(1993) & $\mathrm{SC}$ & $\begin{array}{l}1988,1989, \\
1990\end{array}$ \\
\hline $\begin{array}{l}\text { Boreas NSA-Fen, } \\
\text { Canada }\end{array}$ & $55^{\circ} 55^{\prime} \mathrm{N}, 98^{\circ} 25^{\prime} \mathrm{W}$ & Bubier et al. (1998) & $\mathrm{SC}$ & 1994,1996 \\
\hline Salmisuo, Finland & $62^{\circ} 47^{\prime} \mathrm{N}, 30^{\circ} 56^{\prime} \mathrm{E}$ & Saarnio et al.( 1997) & $\mathrm{SC}$ & 1993 \\
\hline Degeröe, Sweden & $64^{\circ} 11^{\prime} \mathrm{N}, 19^{\circ} 33^{\prime} \mathrm{E}$ & Granberg et al. (2001) & $\mathrm{SC}$ & $\begin{array}{l}1995,1996, \\
1997\end{array}$ \\
\hline Alaska, USA & $64^{\circ} 52^{\prime} \mathrm{N}, 147^{\circ} 51^{\prime} \mathrm{W}$ & $\begin{array}{l}\text { Whalen and Reeburgh } \\
\text { (1992) }\end{array}$ & $\mathrm{SC}$ & $\begin{array}{l}1987,1988, \\
1989,1990\end{array}$ \\
\hline Stordalen, Sweden & $68^{\circ} 20^{\prime} \mathrm{N}, 19^{\circ} 03 \mathrm{E}$ & Svensson et al. (1999) & $\mathrm{SC}$ & $\begin{array}{l}1974,1994, \\
1995\end{array}$ \\
\hline $\begin{array}{l}\text { Boreas SSA-Fen, } \\
\text { Canada }\end{array}$ & $53^{\circ} 48^{\prime} \mathrm{N}, 104^{\circ} 37^{\prime} \mathrm{W}$ & Verma et al.(1998) & $\mathrm{EC}$ & 1994,1995 \\
\hline Stordalen, Sweden & $68^{\circ} 20^{\prime} \mathrm{N}, 19^{\circ} 03 \mathrm{E}$ & $\begin{array}{l}\text { Jackowicz-Korczynski et } \\
\text { al. (2010) }\end{array}$ & $\mathrm{EC}$ & 2006,2007 \\
\hline $\begin{array}{l}\text { Alaska-BEO1, } \\
\text { USA }\end{array}$ & $71^{\circ} 17^{\prime} \mathrm{N}, 156^{\circ} 37^{\prime} \mathrm{W}$ & $\begin{array}{l}\text { Raz-Yaseef, N. } \\
\text { (NGEE-Arctic) }\end{array}$ & $\mathrm{EC}$ & 2012,2013 \\
\hline $\begin{array}{l}\text { Alaska-BEO2, } \\
\text { USA }\end{array}$ & $71^{\circ} 17^{\prime} \mathrm{N}, 156^{\circ} 37^{\prime} \mathrm{W}$ & Zona et al., (2016) & $\mathrm{EC}$ & 2013,2014 \\
\hline Alaska-BES, USA & $71^{\circ} 17^{\prime} \mathrm{N}, 156^{\circ} 36^{\prime} \mathrm{W}$ & Zona et al., (2016) & $\mathrm{EC}$ & 2013,2014 \\
\hline $\begin{array}{l}\text { Alaska-CMDL, } \\
\text { USA }\end{array}$ & $71^{\circ} 19^{\prime} \mathrm{N}, 156^{\circ} 37^{\prime} \mathrm{W}$ & Zona et al., (2016) & EC & 2013,2014 \\
\hline Alaska-ATQ, USA & $70^{\circ} 28^{\prime} \mathrm{N}, 157^{\circ} 25^{\prime} \mathrm{W}$ & Zona et al., (2016) & $\mathrm{EC}$ & 2013,2014 \\
\hline Alaska-IVO, USA & $68^{\circ} 29^{\prime} \mathrm{N}, 155^{\circ} 45^{\prime} \mathrm{W}$ & Zona et al., (2016) & $\mathrm{EC}$ & 2013,2014 \\
\hline Alaska-FAI, USA & $64^{\circ} 52^{\prime} \mathrm{N}, 147^{\circ} 51^{\prime} \mathrm{W}$ & Iwata et al., (2015) & $\mathrm{EC}$ & $\begin{array}{l}2011,2012, \\
2013\end{array}$ \\
\hline Alaska-IMN, USA & $68^{\circ} 37^{\prime} \mathrm{N}, 149^{\circ} 18^{\prime} \mathrm{W}$ & $\begin{array}{l}\text { EUSKIRCHEN, E. S. } \\
\text { (UAF) }\end{array}$ & $\mathrm{EC}$ & $\begin{array}{l}2012,2013, \\
2014\end{array}$ \\
\hline
\end{tabular}

Table S1. Summary of the sites and measurements used in comparison with model prediction. SC: static chamber; EC: eddy covariance tower. 


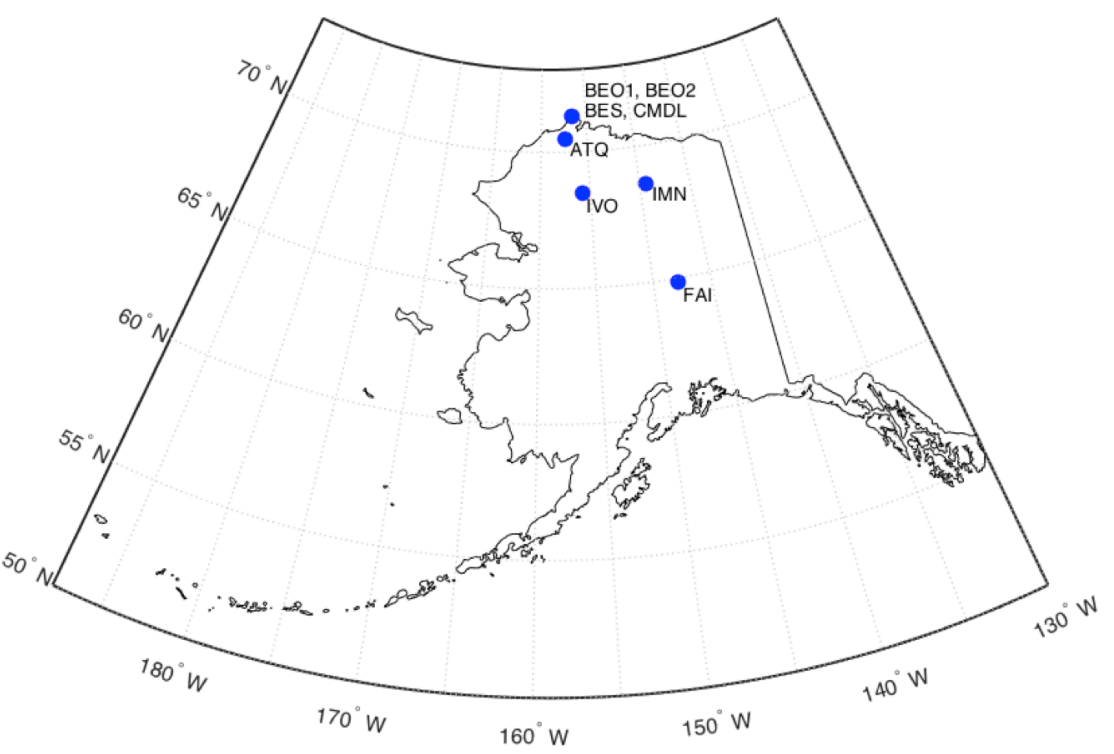

Fig. S2 The eight eddy covariance (EC) sites in Alaska 
Table S2. The flight dates of CARVE measurements in 2012 and 2013

\begin{tabular}{|c|c|c|}
\hline Year & Month & Dates \\
\hline \multirow{6}{*}{2012} & May & $23,24,27,28,30$ \\
\hline & June & $1,18,19,21,22,24$ \\
\hline & July & $17,22,24,25$ \\
\hline & August & $14,18,19,20,21,22,23$ \\
\hline & September & $17,18,19,21,22,23,24,26$ \\
\hline & October & 1 \\
\hline \multirow{7}{*}{2013} & April & $2,3,4,5,6$ \\
\hline & May & $2,4,6,7,8,9,10,13$ \\
\hline & June & $2,3,6,7,8,9,11$ \\
\hline & July & $3,4,5,7,9,11,12$ \\
\hline & August & $2,3,4,7,11,12,13$ \\
\hline & September & $5,6,7,10,12$ \\
\hline & October & $24,25,26,27$ \\
\hline
\end{tabular}

